\title{
Reprodutibilidade do método Cobb na medição da lordose lombar de crianças utilizando diferentes níveis vertebrais
}

\author{
Reproducibility of Cobb method in measureming lumbar lordosis in \\ children using different vertebral levels
}

\section{Juliana Adami Sedrez \\ Bórbara Vendramini Marchetti ${ }^{2}$ \\ Eduardo Gonçalves Raupp 3 \\ Bruna Nichele da Rosa ${ }^{4}$ \\ Rafael Paiva Ribeiro ${ }^{5}$ \\ Clóudia Tarragô Candotti ${ }^{6}$}

Endereço para Correspondência:

Bruna Nichele da Rosa

Rua Diomário Moojen, 20, apartamento 205. Bairro Cristal 90820-030 - Porto Alegre, RS [Brasil]

bruna.nichele@gmail.com
1 Programa de Pós-Graduação em Ciências do Movimento Humano da Universidade Federal do Rio Grande do Sul UFRGS. Porto Alegre, RS - Brasil.

ORCID: https://orcid.org/0000-0003-4933-440X

2 Programa de Pós-Graduação em Ciências do Movimento Humano da Universidade Federal do Rio Grande do Sul UFRGS. Porto Alegre, RS - Brasil.

ORCID: https://orcid.org/0000-0002-1099-8586

3 Programa de Pós-Graduação em Ciências do Movimento Humano da Universidade Federal do Rio Grande do Sul UFRGS. Porto Alegre, RS - Brasil.

ORCID: https://orcid.org/0000-0003-2716-7065

4 Programa de Pós-Graduação em Ciências do Movimento Humano da Universidade Federal do Rio Grande do Sul UFRGS. Porto Alegre, RS - Brasil.

ORCID: https://orcid.org/0000-0003-4590-0654

5 Programa de Pós-Graduação em Ciências do Movimento Humano da Universidade Federal do Rio Grande do Sul UFRGS. Porto Alegre, RS - Brasil.

ORCID: https://orcid.org/0000-0002-2696-9026

6 Cursos de Fisioterapia e Educação Física, e Programa de Pós-Graduação em Ciências do Movimento Humano da Universidade Federal do Rio Grande do Sul - UFRGS. Porto Alegre, RS - Brasil.

ORCID: https://orcid.org/0000-0002-8676-9157

\section{Resumo}

Introdução: A radiografia é o padrão ouro para avaliação da coluna vertebral. Contudo, apresenta uma variabilidade inerente ao exame e ao avaliador. Objetivos: (1) comparar os ângulos de lordose lombar utilizando diferentes níveis vertebrais de referência e (2) verificar a repetibilidade e a reprodutibilidade do método $\mathrm{Cobb}$ na avaliação da lordose lombar de crianças. Métodos: Participaram do estudo 50 crianças, idade de 11,5 $\pm 2,6$ anos. Os exames foram avaliados de forma independente por três avaliadores utilizando os níveis vertebrais L1-L5 e L1-S1. Os dados foram analisados com ICC, SEM e MDC $(\alpha<0,05)$. Resultados: Os ângulos Cobb, usando L1-L5, são de $11^{\circ}$ a $13^{\circ}$ menores que aqueles utilizando L1-S1. Os ICCs foram excelentes para repetibilidade e reprodutibilidade intra-avaliador, e de excelentes (L1-L5) a moderados (L1-S1), para a reprodutibilidade interavaliador. Conclusão: Ambas as metodologias são passíveis de serem utilizadas na avaliação da lordose lombar, pelo mesmo avaliador, de crianças e adolescentes. Ainda, é de fundamental importância que o avaliador saiba das diferenças entre os níveis de referência disponíveis.

Descritores: Reprodutibilidade dos testes. Criança. Região lombossacral. Estudos de avaliação como assunto. Coluna vertebral.

\begin{abstract}
Introduction: Radiography is the gold standard for spinal evaluation. However, there is inherent variability in the test and the evaluator. Objectives: (1) to compare lumbar lordosis angles using different vertebral reference levels (2) to verify the repeatability and reliabilities of the Cobb method for assessment of lumbar lordosis in children. Methods: Fifty children, aged $11.5 \pm 2.6$ years, participated in the study. The exams were evaluated independently by three evaluators, using the vertebral levels L1-L5 and L1-S1. The data were analyzed using ICC, SEM and MDC $(\alpha<0.05)$. Results: The Cobb angles, using L1-L5, are $11^{\circ}$ to $13^{\circ}$ lower than those using L1-S1. ICCs were excellent for repeatability and intrarater reliability and ranged from excellent (L1-L5) to moderate (L1-S1) for inter-rater reliability. Conclusion: Both methodologies can be used by the same evaluator in the assessment of lumbar lordosis of children and adolescents. Furthermore, it is of fundamental importance that the evaluator know the differences between the available reference levels.
\end{abstract}

Keywords: Reproducibility of results. Child. Lumbosacral region. Evaluation studies as topic. Spine. 


\section{Introdução}

As alterações posturais são disfunções que afetam crianças e adolescentes, sendo, nessa população, alvo de grande preocupação, não só pelas elevadas taxas encontradas ${ }^{1}$, mas também pela possibilidade de instalação destas ao longo dos anos ${ }^{2}$, prejudicando o crescimento normal ${ }^{3}$. Além disso, essas alterações têm sido consideradas como fator predisponente às condições degenerativas na fase adulta ${ }^{4}$. Nessa perspectiva, a avaliação postural, principalmente a da coluna vertebral, durante a infância e adolescência é importante para diagnosticar essas alterações precocemente.

A radiografia é o padrão ouro na avaliação da coluna vertebral, com a utilização do método Cobb para o cálculo das medidas angulares, uma vez que, a partir desse exame, o tratamento e o acompanhamento da evolução da alteração podem ser determinados 5 . Contudo, tem sido descrito uma variabilidade inerente ao exame e ao avaliador, seja na obtenção da radiografia, ou na análise desta, o que pode acarretar em um erro de medida de até 5 graus $^{6}$. Ademais, até onde se tem conhecimento, nos estudos disponíveis em que se analisam a reprodutibilidade e as características psicométricas do método de Cobb na avaliação da lordose lombar, utilizamse radiografias de adultos ${ }^{7-10}$. A coluna vertebral de crianças não é idêntica a de adultos, seja concernente aos valores de referências para normalidade da curvatura ${ }^{11}$, como à maturação óssea. De fato, durante a infância a maturação óssea ainda não está completa, o que pode tornar difícil a avaliação radiológica em decorrência da dificuldade de visualização dos pontos anatômicos de referência necessários para mensuração do método de $\mathrm{Cobb}^{12}$. Nesse contexto, avaliar a reprodutibilidade do método de Cobb, bem como o erro do avaliador inerente ao método, na mensuração das curvaturas de crianças e adolescentes é um passo essencial na investigação precoce das alterações posturais.

Não obstante, devem-se considerar as opções na escolha dos níveis vertebrais usados como referência para a realização dos cálculos angulares?. Com relação à lombar, alguns autores consideram apenas a curvatura lombolombar (L1-L5) para mensuração da lordose ${ }^{13}$, enquanto outros associam a primeira vértebra sacral (S1), utilizando assim a curvatura lombossacra (L1-S1) nessa medida ${ }^{7,13,14}$. Ainda, o uso de um ou outro nível vertebral também pode contribuir para as discrepâncias existentes na literatura acerca dos valores de normalidade do ângulo Cobb para a lordose lombar ${ }^{7,13,14}$.

Uma recente revisão sistemática com metanálise ${ }^{11}$ mostra que os ângulos de Cobb, oriundos da avaliação da lordose lombar pelos níveis L1-L5 e L1-S1, têm uma diferença média de $8^{\circ}$ para as crianças, e de $13^{\circ}$ para os adolescentes. Ou seja, na avaliação da lordose lombar dessa população, ao utilizar a região sacral como um dos níveis vertebrais de base, os ângulos seriam superestimados em relação à mensuração da curvatura lombo-lombar. Além disso, maiores ângulos de lordose lombar estão correlacionados com uma maior horizontalização sacral; e o inverso também é verdadeiro, ou seja, menores ângulos estão correlacionados com uma verticalização sacral ${ }^{15,16}$. Para mais, alguns autores relatam que a inclinação sacral não muda após os 3 anos de idade $e^{17,18}$, ao contrário da cifose torácica que aumenta durante a infância ${ }^{18,19}$. Apesar das evidências sobre as diferenças existentes na mensuração da lordose lombar ao utilizar diferentes níveis vertebrais de referência, não há um consenso sobre qual nível vertebral é o mais adequado para essa avaliação. Dessa forma, acredita-se que a avaliação da reprodutibilidade e das demais características psicométricas do método de Cobb (como erro padrão de medida e mínimo erro detectável) poderia auxiliar na escolha do melhor nível vertebral, ao identificar qual apresenta melhor reprodutibilidade e menores erros de medida.

Nessa perspectiva, ao avaliar as propriedades psicométricas do método Cobb na avaliação da lordose lombar de crianças e adolescentes, considerando a influência do avaliador e a escolha dos níveis vertebrais limites da curvatura, é 
possível identificar se esse método pode ser utilizado para monitorar as progressões das alterações posturais, até mesmo por profissionais diferentes sem perder a qualidade das informações obtidas. Assim, os objetivos neste estudo são: (1) comparar os ângulos de lordose lombar utilizando diferentes níveis vertebrais de referência e (2) avaliar a repetibilidade e a reprodutibilidade intra e interavaliador do método Cobb para avaliação da lordose lombar de crianças e adolescentes.

\section{Métodos}

A amostra foi consecutiva, e o tamanho amostral foi calculado assumindo-se que o valor da hipótese nula para o Coeficiente de Correlação Intraclasse (ou Intraclass Correlation Coefficient - ICC) era de 0,6 (ou seja, qualquer valor menor de 0,6 seria considerado inaceitável); $80 \%$ de poder; duas e três medições replicadas (duas vezes com o mesmo avaliador e uma vez com cada avaliador); e um nível de significância de $95 \%$ para detectar o valor ICC de pelo menos $0,8^{20}$, a amostra mínima precisaria consistir de 39 indivíduos. Considerando eventuais perdas, foram convidados 50 participantes. Os sujeitos elegíveis deveriam preencher os seguintes critérios de inclusão: ter idade entre 6 e 13 anos, poder permanecer em ortostase sem auxílio e apresentar requisição médica para a realização de radiografia da coluna vertebral. Foram excluídas as crianças submetidas à cirurgia prévia na coluna vertebral, com alguma alteração ortopédica, com presença de dor no momento da avaliação ou com qualquer anomalia congênita.

Os indivíduos que concordaram em participar do estudo e cujos pais ou responsáveis assinaram o Termo de Consentimento Livre Esclarecido foram incluídos nas análises. O estudo foi aprovado pelo Comitê de Ética em Pesquisa da Universidade onde foi realizado, sob o número CAAE 31062314.5.0000.5347 e respeitou a Resolução 196/96 do Conselho Nacional de Saúde. Os exames radiológicos digitais e panorâmicos, na incidência perfil direito, foram realiza- dos pela mesma equipe técnica de um hospital da cidade de Porto Alegre (RS), a fim de que todas as radiografias seguissem o mesmo protocolo de avaliação. Foram utilizados exames realizados entre os meses de julho e novembro de 2012.

Cada participante foi submetido a apenas uma radiografia. Portanto, a mesma radiografia foi avaliada de forma independente por um quiropraxista (Avaliador A) e dois fisioterapeutas (Avaliadores B e C), todos experientes na área de ortopedia. Cada profissional avaliou o mesmo exame três vezes, sendo duas em instantes sucessivos (Avaliações 1,2) e uma com uma semana de intervalo (Avaliação 3). As avaliações foram realizadas com o método de Cobb modificado de duas linhas ${ }^{6}$, utilizando uma rotina desenvolvida no software Matlab 7.9, para digitalização dos pontos anatômicos de interesse e cálculo do ângulo de lordose lombar. O método de Cobb modificado de duas linhas mensura a curvatura lombar pelo ângulo entre as duas linhas retas tangentes ao platô vertebral superior e inferior das suas respectivas vértebras finais ${ }^{6}$ (Figura 1). O ângulo de lordose lombar foi calculado a partir de dois níveis vertebrais: (a) limite superior de referência como o platô superior de L1 e como limite inferior utilizou-se o platô inferior de L5 e (b) platô superior de L1 e o platô superior de S1 (Figura 1).

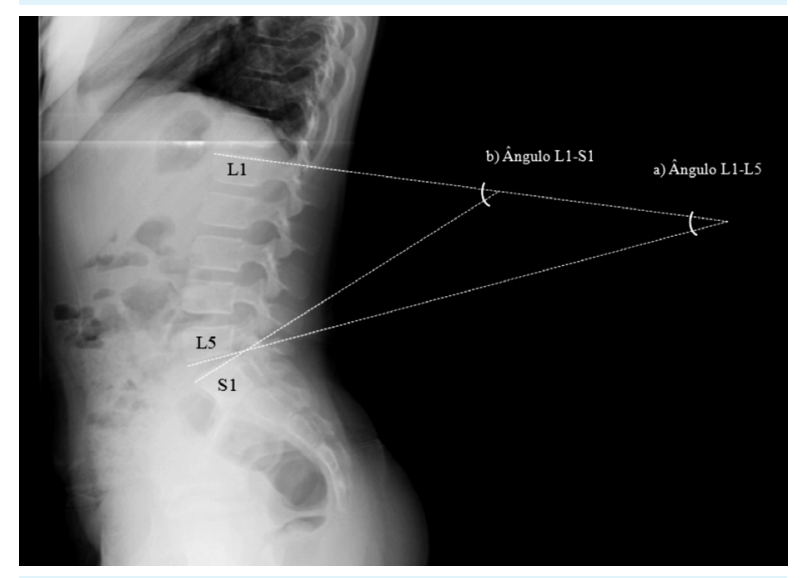

Figura 1: Cálculo da lordose lombar pelo método Cobb modificado de duas linhas ${ }^{6}$ utilizando os limites: a) lombo-lombar (Ll-L5), e b) lombossacra (Ll-Sl)

Fonte: Os autores com base nos dados da pesquisa. 


\section{Anoúlise estatística}

Foi confirmada a normalidade dos dados por meio do teste de Shapiro-Wilk e foram realizadas análises descritivas, com média e desvio-padrão. A fim de identificar a diferença no ângulo de lordose lombar entre as avaliações de dias diferentes (Avaliação $1 \times$ Avaliação 2, para repetibilidade; Avaliação 1 x Avaliação 3, para reprodutibilidade intra-avaliador) foi realizado o teste $t$ de Student pareado. Para verificar a diferença no ângulo da lordose lombar entre os três avaliadores (A, B e C) foi realizada uma ANOVA One Way para cada nível vertebral testado (L1-L5 e L1-S1). Para verificar a diferença do ângulo de lordose lombar entre os níveis vertebrais de referência em cada uma das avaliações foi realizado o teste t pareado.

Para avaliação da reprodutibilidade do método de Cobb foram seguidas as recomendações do Consensus-Based Standards for the Selection of Health Status Measurement Instruments $(\text { COSMIN })^{21}$. Foram calculados: o coeficiente de correlação intraclasse (Intraclass Correlation Coefficient - ICC); o alfa de Cronbach; o erro padrão da medição (Standard Error of Measurement -SEM) e a mínima mudança detectável (Minimal Detectable Change - MDC). O SEM representa o erro de medição, descrito na unidade de medida real, e foi calculado com base na fórmula $S E M=d p \sqrt{1-I C C}$, sendo o $d p$ o desvio-padrão do conjunto de resultados observados ${ }^{22,23}$. A MDC refere-se à quantidade mínima de mudança que reflete a mudança real do paciente, desconsiderando o erro da medida, dada pela fórmula $M D C=e$ score $-z \times d p \times \sqrt{2(1-I C C)} 22,23$.

A repetibilidade foi avaliada a partir do ICC obtido entre as duas avaliações sucessivas (Avaliação 1 e 2) do mesmo avaliador, enquanto a reprodutibilidade intra-avaliador foi avaliada a partir do ICC entre a primeira avaliação e outra uma semana depois (Avaliação 1 e 3) do mesmo avaliador. A repetibilidade e a reprodutibilidade intra-avaliador foram testadas para os três avaliadores. A reprodutibilidade interavaliador foi dada pelo ICC obtido entre a primeira avaliação (Avaliação 1) dos três avaliadores (Avaliador
A, B e C). Os valores de ICC foram classificados como fraco (ICC $<0,40)$, moderado (ICC entre $0,4$ e 0,75$)$ e excelente (ICC $>0,75$ ) de acordo com Shrout e Fleisss ${ }^{24}$. Para o alfa de Cronbach, valores acima de 0,7 são considerados como ideais ${ }^{25}$. Foi utilizado o nível de significância de 0,05 em todos os testes estatísticos, realizados no software SPSS, versão 20.0.

\section{Resultados}

As crianças que participaram do estudo tinham média de idade de 11,5 $\pm 2,6$ anos, massa corporal de 42,9 $\pm 15,2 \mathrm{~kg}$ e estatura de $143 \pm 15,2$ $\mathrm{cm}$. A Tabela 1 apresenta os resultados da repetibilidade e reprodutibilidade intra-avaliador para a avaliação da lordose lombar a partir dos dois níveis vertebrais testados (L1-L5 e L1-S1). Observam-se excelente reprodutibilidade e alta consistência interna do método de Cobb para mensuração da lordose lombar utilizando-se os dois níveis vertebrais. Também foram obtidos baixos valores de SEM e MDC.

A Tabela 2 apresenta os resultados de reprodutibilidade interavaliador para a avaliação da lordose lombar por meio do método de Cobb para os dois níveis vertebrais testados (L1-L5 e L1-S1). Foram observados alta consistência interna de ambos os níveis vertebrais testados bem como baixos valores de SEM. Ao utilizar o nível vertebral L1-L5, encontrou-se excelente reprodutibilidade interavaliador. Para o nível L1-S1 obteve-se moderada reprodutibilidade interavaliador. Contudo, houve diferença significativa entre os avaliadores para ambos os níveis vertebrais.

\section{Discussão}

Os resultados apontam que a repetibilidade e a reprodutibilidade intra-avaliador do método de Cobb (Tabela 1) são excelentes (ICC > $0,91 ; \mathrm{p}<0,001)$, com baixo SEM $\left(2^{\circ}\right.$ a $\left.3^{\circ}\right)$ e MDC $\left(4^{\circ}\right.$ a $\left.6,5^{\circ}\right)$, não existindo diferença significativa 
Tabela l: Resultados de repetibilidade e reprodutibilidade intra-avaliador das medidas do ângulo de Cobb de lordose lombar, utilizando os diferentes níveis vertebrais

\begin{tabular}{|c|c|c|c|c|c|c|c|c|}
\hline \multicolumn{9}{|c|}{ Repetibilidade (avaliações sucessivas) } \\
\hline & $\begin{array}{c}1^{\circ} \text { avaliação } \\
\text { Média (DP) } \\
\left({ }^{\circ}\right)\end{array}$ & $\begin{array}{c}2^{\circ} \text { avaliação } \\
\text { Média (DP) } \\
\left({ }^{\circ}\right)\end{array}$ & $\begin{array}{c}\mathrm{p} \\
\text { (Teste t) }\end{array}$ & ICC (IC 95\%) & $\mathrm{p}$ & $\begin{array}{c}\text { Alfa de } \\
\text { Cronbach }\end{array}$ & $\begin{array}{c}\text { SEM } \\
\left({ }^{\circ}\right)\end{array}$ & $\begin{array}{c}\text { MDC } \\
\left({ }^{\circ}\right)\end{array}$ \\
\hline \multicolumn{9}{|c|}{ Lordose lombar (L1-L5) } \\
\hline Avaliador A & $35,7 \pm 11,9$ & $35 \_ \pm 11,6$ & 0,057 & $|0,97(0,95-0,98)|$ & $<0,001^{* *}$ & 0,98 & 2 & 4 \\
\hline Avaliador B & $40,1 \pm 12,8$ & $40,3 \pm 13,4$ & 0,803 & $0,96(0,93-0,98)$ & $<0,001^{* *}$ & 0,98 & 2,6 & 5 \\
\hline Avaliador C & $43,8 \pm 13,9$ & $44,1 \pm 13,9$ & 0,538 & $0,97(0,96-0,98)$ & $<0,001^{* *}$ & 0,98 & 2,4 & 5 \\
\hline \multicolumn{9}{|c|}{ Lordose lombar (L1-S1) } \\
\hline Avaliador A & $41,3 \pm 11$ & $47,4 \pm 11,8$ & 0,909 & $|0,96(0,93-0,97)|$ & $<0,001^{* *}$ & 0,98 & 2,3 & 4,5 \\
\hline Avaliador B & $51,9 \pm 12,3$ & $52 \pm 12,2$ & 0,762 & $0,95(0,92-0,97)$ & $<0,001^{* *}$ & 0,97 & 2,7 & 5 \\
\hline Avaliador C & $57,3 \pm 13,8$ & $56,5 \pm 13,4$ & 0,220 & $0,94(0,9-0,96)$ & $<0,001^{* *}$ & 0,97 & 3,3 & 6,5 \\
\hline $\begin{array}{c}\text { Diferença } \\
\text { média entre } \\
\text { níveis (IC95\%) }\end{array}$ & $\begin{array}{c}12,2 \\
(9,2-15,2)^{*}\end{array}$ & $\begin{array}{c}12,7 \\
(9,6-15,7) *\end{array}$ & & & & & & \\
\hline
\end{tabular}

Reprodutibilidade intra-avaliador (intervalo de uma semana entre as avaliações)

\begin{tabular}{|c|c|c|c|c|c|c|c|c|}
\hline & $\mid \begin{array}{c}1^{\circ} \text { avaliação } \\
\text { Média (DP) } \\
\left({ }^{\circ}\right)\end{array}$ & $\begin{array}{c}3^{\circ} \text { avaliação } \\
\text { Média (DP) } \\
\left({ }^{\circ}\right)\end{array}$ & $\begin{array}{c}p \\
\text { (Teste t) }\end{array}$ & ICC (IC95\%) & $\mathrm{p}$ & $\begin{array}{c}\text { Alfa de } \\
\text { Cronbach }\end{array}$ & $\begin{array}{c}\text { SEM } \\
\left({ }^{\circ}\right)\end{array}$ & $\begin{array}{c}\mathrm{MDC} \\
\left({ }^{\circ}\right)\end{array}$ \\
\hline \multicolumn{9}{|c|}{ Lordose lombar (L1-L5) } \\
\hline Avaliador A & $35,7 \pm 11,9$ & $33,3 \pm 11,7$ & 0,283 & $|0,97(0,96-0,98)|$ & $<0,001^{* *}$ & 0,98 & 2 & 4 \\
\hline Avaliador B & $40,2 \pm 12,8$ & $42,4 \pm 13,3$ & $<0,001$ & $0,96(0,93-0,97)$ & $<0,001^{* *}$ & 0,98 & 3 & 5 \\
\hline Avaliador C & $43,8 \pm 13,9$ & $43 \pm 14,9$ & 0,382 & $0,95(0,91-0,97)$ & $<0,001^{* *}$ & 0,97 & 3 & 6 \\
\hline \multicolumn{9}{|c|}{ Lordose lombar (L1-S1) } \\
\hline Avaliador A & $47,3 \pm 11$ & $47,8+11,3$ & 0,396 & $|0,95(0,92-0,97)|$ & $<0,001^{* *}$ & 0,97 & 2 & 5 \\
\hline Avaliador B & $51,9 \pm 12,3$ & $53,6 \pm 11,5$ & $<0,001$ & $0,95(0,92-0,97)$ & $<0,001^{* *}$ & 0,97 & 3 & 5 \\
\hline Avaliador C & $57,3 \pm 13,8$ & $58 \pm 12,8$ & 0,356 & $0,91(0,85-0,95)$ & $<0,001^{* *}$ & 0,95 & 4 & 8 \\
\hline $\begin{array}{c}\text { Diferença } \\
\text { média entre } \\
\text { níveis (IC95\%) }\end{array}$ & $\begin{array}{c}12,2 \\
(9,2-15,2)^{*}\end{array}$ & $\begin{array}{c}12,8 \\
(9,8-15,8)^{*}\end{array}$ & & & & & & \\
\hline
\end{tabular}

${ }^{*} p<0,001$ a partir do teste t pareado ** Correlação significativa; + Diferença significativa entre as médias Fonte: Os autores com base nos dados da pesquisa, 2012.

Tabela 2: Resultados de reprodutibilidade interavaliador da medição do ângulo de Cobb de lordose lombar

\begin{tabular}{|c|c|c|c|c|c|c|c|c|}
\hline $\begin{array}{l}\text { Lordose } \\
\text { Lombar }\end{array}$ & $\begin{array}{c}\text { Avaliador A } \\
\text { Média (DP) } \\
\left({ }^{\circ}\right)\end{array}$ & $\begin{array}{c}\text { Avaliador B } \\
\text { Média (DP) } \\
\left({ }^{\circ}\right)\end{array}$ & $\begin{array}{c}\text { Avaliador C } \\
\text { Média (DP) } \\
\left({ }^{\circ}\right)\end{array}$ & $\begin{array}{c}p \\
\text { (ANOVA) }\end{array}$ & ICC (IC 95\%) & $\begin{array}{c}\text { Alfa de } \\
\text { Cronbach }\end{array}$ & $\begin{array}{l}\text { SEM } \\
\left({ }^{\circ}\right)\end{array}$ & $\begin{array}{c}\mathrm{MDC} \\
\left(^{\circ}\right)\end{array}$ \\
\hline L1-L5 & $35,7 \pm 11,9$ & $40,1 \pm 12,8$ & $43,8 \pm 13,9$ & $0,009^{+}$ & $0,85(0,73-0,85)^{* *}$ & 0,92 & 6 & 12 \\
\hline L1-S1 & $47,3 \pm 11$ & $51,9 \pm 12,3$ & $57,3 \pm 13,8$ & $<0,001^{+}$ & $0,74(0,62-0,83)^{* *}$ & 0,89 & 7 & 13 \\
\hline $\begin{array}{c}\text { Diferença } \\
\text { média entre } \\
\text { níveis vertebrais } \\
\text { (IC 95\%) }\end{array}$ & $\begin{array}{l}11,5(6,5 \\
-16,5)^{*}\end{array}$ & $\begin{array}{l}11,7(6,7 \\
-16,7)^{*}\end{array}$ & $\begin{array}{l}13,4(8,4 \\
-18,4)^{*}\end{array}$ & & & & & \\
\hline
\end{tabular}


entre os avaliadores. Também destacam-se os baixos valores de MDC, mostrando que, a partir de $6^{\circ}$ de diferença entre exames, já pode-se considerar alteração real do paciente.

Para a reprodutibilidade interavaliador (Tabela 2), apesar de o ICC ser excelente para L1-L5, e moderado para L1-S1, encontrou-se diferença significativa entre todos os avaliadores $(\mathrm{AxB} p=0,04 ; \mathrm{AxC} \mathrm{p}=0,005 ; \mathrm{BxC} \mathrm{p}=0,036)$, independentemente do nível vertebral avaliado. Os valores elevados de MDC $\left(11,8^{\circ}\right.$ para L1-L5; e $13,4^{\circ}$ para LI-S1) sugerem que, com diferentes avaliadores, eleva-se o erro de medição, e, com isso, aumenta-se a diferença mínima necessária para ser considerada alteração real do paciente.

Corroborando o estudo aqui apresentado, Polly et al. ${ }^{10}$ obtiveram excelente reprodutibilidade intra-avaliador (ICC > 0,87 para LI-L5; ICC > 0,83 para L1-S1), assim como excelente reprodutibilidade interavaliador (ICC > 0,90 para LI-L5; ICC > 0,81 para LI-S1), de três avaliadores: um cirurgião ortopédico de coluna, um ortopedista pediátrico e um radiologista. Porém, esses autores não reportaram SEM ou MDC. O trabalho de Lee et al. ${ }^{9}$, como a atual pesquisa, também mostrou excelente reprodutibilidade intra-avaliador (ICC > 0,95 para LI-L5; ICC > 0,94 para L1-S1) e intervaliador (ICC > 0,95 para LI-L5; ICC > 0,92 para L1-S1), em pacientes com e sem espondilite anquilosante. Em contrapartida, o SEM e a MDC não foram avaliados.

Hicks et al. ${ }^{8}$ testaram a reprodutibilidade interavaliador na avaliação radiográfica da lordose lombo-lombar L1-L5 pela técnica de Cobb e obtiveram ICC $=0,98, \mathrm{SEM}=1,99^{\circ}, \mathrm{MDC}=3,90^{\circ}$. No atual estudo, apesar de ter sido igualmente encontrado um ICC excelente, os resultados da MDC $\left(12^{\circ}\right)$ e do SEM $\left(6^{\circ}\right)$ foram mais elevados. Harrison et al. ${ }^{26}$ apresentaram resultados excelentes para a reprodutibilidade intra e interavaliador (ICC $=0,99$ e 0,98, respectivamente), porém os valores de SEM e MDC não foram mencionados.

Outros autores também incluem nas suas avaliações distintas formas de mensuração ao Cobb, como o método centroide, as tangen- tes posteriores do corpo vertebral e o método TRALL ${ }^{9,26-28}$. Não obstante, o modo de avaliação da coluna lombar mais comumente adotado na prática clínica é o método Cobb, cuja reprodutibilidade tem-se mostrado superior em comparação a outras formas de análise 9 . Além do mais, trabalhos expõem que o ângulo Cobb é sensível a alterações oriundas da osteoporose, tais como cunha, bicôncava e escova ${ }^{10,29}$.

$\mathrm{Na}$ pesquisa atual, a reprodutibilidade para L1-L5 foi maior, e isso é confirmado por investigações anteriores já disponíveis na literatura $^{9,10}$. Extrapolando as características para a população adulta, conforme Polly et al. ${ }^{10}$ relatam, um aspecto negativo da utilização da vértebra S1 é que ela é constantemente influenciada por alterações degenerativas e seu contorno, em geral, torna-se prejudicado em pacientes com artrite. Destaca-se que o atual estudo apresenta resultados excelentes de repetibilidade e reprodutibilidade intra-avaliador (ICC > 0,91; $\mathrm{p}<0,001)$, indicando que avaliações feitas pelo mesmo profissional/clínico, com o método mais comumente utilizado para avaliação da lordose lombar - que é o Cobb -, são confiáveis.

Uma metanálise recente identificou que os valores de referência para a lordose lombar de crianças, utilizando L1-L5 (curvatura lombolombar) e L1-S1 (curvatura lombossacra), são de $34,5^{\circ}$ até $44,8^{\circ}$ e $41,7^{\circ}$ até $54,1^{\circ}$, respectivamente ${ }^{11}$. Ao analisar os resultados da atual investigação, observou-se que os valores de lordose utilizando L1-L5 são de $11^{\circ}$ a $13^{\circ}$ menores que aqueles usando L1-S1. Essa informação é importante, pois, dependendo do caso, o profissional/clínico pode diagnosticar e classificar diferentemente $o$ seu paciente utilizando ambos os níveis. Assim, para o acompanhamento e avaliação pré e pósintervenção de um tratamento postural dos pacientes, é necessário que as mensurações da lordose lombar sejam feitas sempre com os mesmos parâmetros de referência.

Adicionalmente, menciona-se que a principal limitação deste estudo é o range de lordose lombar da amostra analisada, uma vez que não foram incluídas nesta crianças com retificação 
da lordose ou aumento importante da curvatura. Desse modo, sugere-se cautela na condução da avaliação da coluna lombar em tais circunstâncias. Outra limitação envolveu o nível de experiência dos avaliadores, pois não foi testado se avaliadores sem experiência ortopédica, como estudantes, por exemplo, poderiam gerar resultados diferentes. Como recomendações para pesquisas futuras, além de sanar as lacunas deixadas, seria testar a reprodutibilidade também das curvaturas da coluna torácica e cervical de crianças e adolescentes.

\section{Conclusão}

Após análise dos resultados, concluiu-se que tanto a utilização do nível vertebral L1-L5 quanto o uso do L1-S1, na avaliação da lordose lombar de crianças e adolescentes, pelo método Cobb modificado de duas linhas, apresentam excelente repetibilidade e reprodutibilidade intra-avaliador, com baixo valor de MDC (de $4^{\circ}$ a $\left.6,5^{\circ}\right)$. Apesar de a utilização dos níveis vertebrais L1-L5 apresentar resultados estreitamente melhores no que diz respeito à reprodutibilidade, ambas as metodologias (L1-L5 e L1-S1) são confiáveis na avaliação da lordose lombar de crianças e adolescentes, pelo mesmo avaliador sucessivamente ou em ocasiões diferentes. Ainda, é de fundamental importância que o avaliador saiba das peculiaridades da forma de mensuração elencada e das diferenças entre os níveis de referência (L1-L5 e L1-S1) disponíveis para compor o seu diagnóstico e acompanhamento.

\section{Referências}

1. Kasten AP, da Rosa BN, Schmit EFD, Noll M, Candotti CT. Prevalence of postural deviations in the spine in schoolchildren: a systematic review with meta-analysis. J Hum Growth Dev. 2017;27(1).

2. Da Rosa BN, Noll M, Sedrez JA, Furlanetto TS, Candotti CT. Monitoring the prevalence of postural changes in schoolchildren. J Phys Ther Sci. 2016;28(2).
3. Dimeglio A, Canavese F. The growing spine: how spinal deformities influence normal spine and thoracic cage growth. Eur Spine J. 2012;21(1):64-70.

4. Sparrey CJ, Bailey JF, Safaee M, Clark AJ, Lafage V, Schwab F, et al. Etiology of lumbar lordosis and its pathophysiology: a review of the evolution of lumbar lordosis, and the mechanics and biology of lumbar degeneration. Neurosurg Focus. 2014;36(5):E1.

5. Negrini S, Aulisa A. SOSORT guidelines: orthopaedic and rehabilitation treatment of idiopathic scoliosis during growth. Scoliosis J. 2012;7(3):7148-61.

6. Vrtovec T, Pernuš F, Likar B. A review of methods for quantitative evaluation of spinal curvature. Eur Spine J. 2009;18(5):593-607.

7. Damasceno LHF, Cararini SRG, Campos AD, Defino HLA. Lordose lombar: estudo dos valores angulares e da participação dos corpos vertebrais e discos intervertebrais. Acta Ortop Bras. 2006;14(4):193-8.

8. Hicks GE, George SZ, Nevitt MA, Cauley JA, Vogt MT. Measurement of lumbar lordosis: interrater reliability, minimum detectable change and longitudinal variation. J Spinal Disord Tech. 2006;19(7):501-6.

9. Lee JS, Goh TS, Park SH, Lee HS, Suh KT. Radiographic measurement reliability of lumbar lordosis in ankylosing spondylitis. Eur Spine J. 2013;22(4):813-8.

10. Polly DW, Kilkelly FX, McHale KA, Asplund LM, Mulligan M, Chang AS. Measurement of lumbar lordosis. Evaluation of intraobserver, interobserver, and technique variability. Vol. 21, Spine. 1996. p. 1530-6.

11. Furlanetto TS, Sedrez JA, Candotti CT, Loss JF. Reference values for Cobb angles when evaluating the spine in the sagittal plane: a systematic review with meta-analysis. Motricidade. 2018;14(2-3):115-28.

12. Tolo V. The lower extremity. In: Publishers L-R, editor. Pediatric orthopaedics. $3^{\text {rd }}$ ed. Philadelphia; 1996. p. 1047-81.

13. Vialle R, Levassor N, Rillardon L, Templier A, Skalli W, Guigui P. Radiographic analysis of the sagittal alignment and balance of the spine in asymptomatic subjects. J Bone Joint Surg Am. 2005;87(2):260-7.

14. Roussouly P, Gollogly S, Berthonnaud E, Dimnet J. Classification of the Normal Variation in the Sagittal Alignment of the Human Lumbar Spine and Pelvis in the Standing Position. Spine (Phila Pa 1976). 2005;30(3):2005. 
15. Roussouly P, Nnadi C. Sagittal plane deformity: an overview of interpretation and management. Eur Spine J. 2010;19(11):1824-36.

16. Schwab FJ, Patel A, Ungar B, Farcy J, Lafage V. Adult Spinal Deformity - Postoperative Standing Imbalance Assessing Alignment and Planning Corrective Surgery. Spine (Phila Pa 1976). 2010;35(25):2224-31.

17. Mac-Thiong JM, Labelle H, Berthonnaud E, Betz RR, Roussouly P. Sagittal spinopelvic balance in normal children and adolescents. Eur Spine J. 2007;16(2):227-34 .

18. Mac-Thiong JM, Labelle H, Roussouly P. Pediatric sagittal alignment. Eur Spine J. 2011;20 Suppl 5:586-90.

19. Cil A, Yazici M, Uzumcugil A, Kandemir U, Alanay A, Alanay Y, et al. The evolution of sagittal segmental alignment of the spine during childhood. Spine (Phila Pa 1976). 2005;30(1):93-100.

20. Walter SD, Eliasziw M, Donner A. Sample size and optimal designs for reliability studies. Stat Med. 1998;17(Apr 1997):101-10.

21. Mokkink LB, Terwee CB, Knol DL, Stratford PW, Alonso J, Patrick DL, et al. The COSMIN checklist for evaluating the methodological quality of studies on measurement properties: A clarification of its content. BMC Med Res Methodol. 2010;10(22).

22. Haley SM, Fragala-Pinkham MA. Interpreting change scores of tests and measures used in physical therapy. Phys Ther. 2006;86(5):735-43.
23. Bruton A, Conway JH, Holgate ST. Reliability: what is it, and how is it measured. Physiotherapy. 2000;86(2):94-9.

24. Shrout PE, Fleiss JL. Intraclass correlations: uses in assessing rater reliability. Psychol Bull. 1979;86(2):420-8

25. Souza AC de, Alexandre NMC, Guirardello E de B. Propriedades psicométricas na avaliação de instrumentos: avaliação da confiabilidade e da validade. Epidemiol Serv Saúde. 2017;26(3):649-59.

26. Harrison DE, Harrison DD, Cailliet R, Janik T, Holland B. Radiographic Analysis of Lumbar Lordosis. Spine (Phila Pa 1976). 2001;26(11):235-42.

27. Chen Y-L. Vertebral Centriod Measurement of Lumbar Lordosis Compared With the Cobb Technique. 1999. p. 1786-90.

28. Hong JY, Suh SW, Modi HN, Hur CY, Song HR, Park JH. Reliability analysis for radiographic measures of lumbar lordosis in adult scoliosis: a casecontrol study comparing 6 methods. Eur Spine J. 2010;19(9):1551-7.

29. Genant HK, Li J, Wu CY, Shepherd JA. Vertebral fractures in osteoporosis: a new method for clinical assessment. J Clin Densitom. 2000;3(3):281-90. 